\title{
Towards more realistic kinetic models for concentrated solutions and melts
}

\author{
Jiannong Fang*, \\ GEOLEP-ICARE-ENAC, Ecole Polytechnique Fédérale de Lausanne, 1015 \\ Lausanne, Switzerland \\ Alexei Lozinski, Robert G. Owens, \\ LMF-ISE-STI, Ecole Polytechnique Fédérale de Lausanne, 1015 Lausanne, \\ Switzerland
}

\begin{abstract}
In this paper we consider modifications of two kinetic models for concentrated polymer solutions and melts: the simplified uniform (SU) version of a thermodynamically admissible single segment reptation model by Öttinger [H.-C. Öttinger, J. Rheol. 43 (1999) 1461-1493, and J. Fang et al., J. Rheol. 44 (2000) 1293-1317] and the encapsulated FENE dumbbell (EFD) model [R. B. Bird and J. R. Deaguiar, J. Non-Newtonian Fluid Mech. 13 (1983) 149-160]. The two modified models incorporate double reptation, convective constraint release (CCR), and chain stretching. In the modified SU model, orientation and stretching are still modelled in a decoupled way, while in the case of the modified EFD model they are modelled in a coupled way. Quantitative comparisons are made for a number of shear and extensional flows between the predictions of the modified EFD model with those of the SU model, the modified SU model, the original EFD model and a recent simple constitutive model by Marrucci and Ianniruberto [G. Marrucci and G. Ianniruberto, Phil. Trans. R. Soc. Lond. A 361 (2003) 677-687]. The models' predictions are also tested against experimental data for entangled polystyrene solutions.

For steady simple shear flow it is shown that good quantitative agreement with experimental data using physically realistic parameters requires that orientation and stretching be modelled in a coupled way. In particular, predictions with the modified SU model of the steady shear viscometric functions are only accurate with a maximum chain stretch ratio parameter an order of magnitude smaller than is required by the polymer chemistry. The use of the modified EFD model with realistic model parameters leads to better overall fits in all cases with the experimental data than either variant of the SU model or the Marrucci-Ianniruberto model.
\end{abstract}

Key words: Kinetic theory, concentrated solutions, melts, reptation model, encapsulated dumbbell model, CCR, CCR2, DCR 


\section{Introduction}

A widely applied class of molecular-based models for concentrated polymer solutions and polymer melts is based on the reptation picture [5] and the theory of Doi and Edwards [9]. The Doi-Edwards (DE) theory for the rheological behavior of entangled polymers successfully predicts the response to large step-shear strains but fails to predict other nonlinear shear properties, such as the steady-state viscosity, for which the theory gives the prediction of excessive shear thinning. This negative feature leads directly to an intrinsic instability for moderately fast shear flows and has long prevented use of the DE constitutive equation in numerical simulations of complex flows. Many attempts have been made in recent years to improve the situation. Several physical effects have been found to be important for more realistic modelling of nonlinear properties of entangled polymers. These are chain stretching $[26,38,29,30]$, double reptation [39,6,31], convective constraint release (CCR) $[25,17]$, and convective conformation renewal (CCR2) due to flow-induced lengthening of tube segments $[18,19]$. In addition, avoiding the independent alignment approximation (IAA) leads to improved results $[8,7,9,24,35]$. A short summary of these effects can be found in [11]. In many works, CCR and CCR2 are found to be critical for predicting the correct shape of the shear stress curve and removing the inconvenient features of the DE model.

Despite these conceptual breakthroughs, it has proved difficult to develop a complete theory on a coarse-grained level. Mead et al. [28] have developed the "contour-variable" theory that incorporates all the well-established phenomena in rather complicated coupled integral-differential equations. Schieber and co-workers [13-15] have proposed a full-chain stochastic approach suitable for computer simulations, with the advantage of including all the plausible physical ideas in a natural way. Very recently, a new thermodynamically admissible reptation model [34] has been developed under the guidance of a general equation for the nonequilibrium reversible-irreversible coupling (GENERIC), a framework for nonequilibrium thermodynamics [12,36,33]. A detailed evaluation of the recommended "uniform" model on a simplified level (referred to hereafter as the SU model, in which no use is made of the ingredient of anisotropic tube cross sections) has been given for shear and extensional flows by stochastic simulations [11]. The SU model incorporates chain stretching, double reptation and CCR whilst avoiding IAA. It is able to describe qualitatively all the nonlinear rheological properties of linear entangled polymers and is highly competitive with the two alternative molecular-based models (the

\footnotetext{
* Corresponding author.

Email addresses: Jiannong.Fang@epfl.ch (Jiannong Fang), Alexei.Lozinski@epfl.ch (Alexei Lozinski), Robert.0wens@epfl.ch (Robert G. Owens).
} 
contour-variable theory and the full-chain simulation model) cited above. Notably, the SU model is a thermodynamically consistent single-segment theory which has only four structural degrees of freedom. However, for the steadystate shear stress curve, it still predicts a negative value for the rate of change of shear stress with shear rate over intermediate values of the latter, which means that the intrinsic instability remains. As with the models of Mead et al. [28] and Schieber and co-workers [13-15] mentioned above, at high shear rates the steady-state extinction angle approaches zero rather than a non-zero plateau.

In two recent papers Marrucci and Ianniruberto $[19,20]$ proposed models featuring separate evolution equations for the average tube orientation tensor $\mathbf{S}$ and the average stretch ratio $\lambda$. The first of these papers [19], a single-mode constitutive theory known as the double-convection-reptation (DCR) model could account for CCR and chain stretch. The model of the second paper [20] extended that of [19] to a multi-mode situation and included chain-end fluctuations. However, it was observed by Wapperom et al. [42] that at high shear rates (larger than the reciprocal Rouse time) the models could manifest shear-thickening and large chain stretch. Marrucci and Ianniruberto [27] attributed this anomalous response to the decoupling of the equations for $\mathbf{S}$ and $\lambda$ and proposed a simple coupled differential constitutive equation which, it was claimed, would overcome the difficulties described above. This has been substantiated numerically in a very recent paper by Wapperom and Keunings [43]: although the behaviour of the decoupled [19] and coupled [27] models in steady and transient shear and uniaxial extensional flows is very similar and in qualitative agreement with experimental data [2,3], the coupled model avoids the anomalous shear-thickening behaviour of its decoupled cousin. Following Bhatacharjee et al. [2] and Wapperom and Keunings [43] we refer to the coupled model of Marrucci and Ianniruberto henceforth as the coupled DCR-CS model.

In this paper (see Sections 2.2 and 2.3), we consider modifications to both the SU model of Öttinger [34] and the encapsulated FENE dumbbell model of Bird and Deaguiar [4]. In the SU model, the effects of constraint release (double reptation and CCR) are introduced through the diffusion term for the orientation of inner chain segments. In the modified model proposed in the present paper, the diffusion term is reformulated to account, additionally, for CCR2 and thereby respect the new physical insight on CCR proposed by Ianniruberto and Marrucci [19]. A stretching relaxation time dependent upon the velocity gradient due to CCR effects is also introduced. As will become plain in the presentation of the numerical results in Section 3.1, the modified SU model is able to more faithfully predict the viscometric functions for steady simple shear flow than the original SU model. However, since the modified SU model still employs a decoupling approximation for orientation and stretch, to get quantitative agreement with the shear data the maximum chain 
stretch ratio has to be chosen an order of magnitude smaller than the value determined on the basis of the polymer chemistry. In an effort to develop a model featuring full coupling between orientation and stretching we introduce in Section 2.3 a modification to the encapsulated FENE dumbbell model of Bird and Deaguiar [4]. CCR and CCR2 are now accounted for through a redefinition of the characteristic relaxation time $\lambda_{H}$ and anisotropy parameter $\sigma$ which appear in the original model. Results of using the new model with realistic parameter values are presented in Sections 3.1 - 3.4. The inconvenient impact on the parameter choice of decoupling orientation and stretch are now removed and in all the flows considered quantitative (Sections 3.1 and 3.3) and qualitative (Sections 3.2 and 3.4) agreement with experimental data represents an improvement over the two variants of the SU model and the coupled DCR-CS model mentioned in the preceding paragraphs.

\section{Description of the models}

\subsection{A coupled constitutive equation by Marrucci and Ianniruberto [27] (cou- pled DCR-CS model)}

Let $\mathbf{Q}$ denote the end-to-end vector of a subchain between consecutive entanglements and let us introduce the quantity

$$
\mathbf{A}=\frac{\langle\mathbf{Q Q}\rangle}{a^{2}}
$$

where $a$ is the length of a segment under equilibrium conditions. Marrucci and Ianniruberto [27] proposed the following evolution equation for $\mathbf{A}$ :

$$
\frac{d \mathbf{A}}{d t}=\boldsymbol{\kappa} \cdot \mathbf{A}+\mathbf{A} \cdot \boldsymbol{\kappa}^{T}-\frac{f}{\tau}\left(\mathbf{A}-\frac{1}{3} \operatorname{tr} \mathbf{A} \boldsymbol{\delta}\right)-\frac{1}{3 \tau_{R}}(f \operatorname{tr} \mathbf{A}-1) \boldsymbol{\delta},
$$

where the orientational relaxation time $\tau$ satisfies

$$
\frac{1}{\tau}=\frac{2}{\tau_{d}}+\left(\frac{1}{\tau_{R}}-\frac{2}{\tau_{d}}\right) \frac{\beta(f \operatorname{tr} \mathbf{A}-1)}{1+\beta(f \operatorname{tr} \mathbf{A}-1)},
$$

and

$$
f=\frac{b-1}{b-\operatorname{tr} \mathbf{A}},
$$

is the finite extensibility factor in the spring force $\mathbf{F}$ in a segment. $\tau_{d}$ and $\tau_{R}$ in (3) denote, respectively, the reptation time and the Rouse time and in (4) $b$ is the maximum value of $\operatorname{tr} \mathbf{A}$. The parameter $\beta$ appearing in (3) is a numerical parameter of order unity and measuring CCR effectiveness [17]. We note in (2) that the first two terms on the right-hand side represent the rate of 
change of $\mathbf{A}$ under affine deformation, whereas the third and fourth groups of terms are concerned with orientation and stretch relaxation. In the expression (3) for the orientational relaxation time, $\tau$ is allowed to vary with shear rate. Thus from $(3)$ we see that for slow flows $(\operatorname{tr} \mathbf{A} \approx 1), \tau \approx \tau_{d} / 2$, this being the disengagement time due to reptation and constraint release. For fast flows, the CCR mechanism sweeps away constraints and reduces the orientational time yet further. From (3) $\tau$ may be seen to approach the Rouse time $\tau_{R}$ for $\operatorname{tr} \mathbf{A} \gg 1$.

The stress $\boldsymbol{\tau}$ for the coupled DCR-CS model is given by

$$
\boldsymbol{\tau}=G f \mathbf{A}
$$

where $G=3 \nu_{1} k_{B} T$ and $\nu_{1}$ is the segment density.

\subsection{Modified SU model}

In order to describe the stochastic effects involved in the dynamics of the primitive chain and the relaxation mechanisms in the SU model, we introduce a configurational distribution function which, for nonhomogeneous flows, depends on the space coordinates $\mathbf{r}$. Well-established configurational variables appearing in the configurational distribution function of reptation models are a unit vector $\mathbf{u}$ and a label $s \in[0,1]$, where $\mathbf{u}$ specifies the orientation of the tube segment or the chain segment inside at the position $s$ of the primitive chain. The values $s=0$ and $s=1$ correspond to the chain ends, and we assume that $s$ varies proportionally to the contour length measured from one of the chain ends. We will keep this labelling rule, including the values 0 and 1 at the chain ends, even in the presence of chain stretching. To this end, we here consider the configurational distribution function $\psi(\mathbf{u}, s, \mathbf{r}, t)$. At any position $\mathbf{r}$ and time $t$, we hence have a probability density in the configurational variables $\mathbf{u}$ and $s$ which gives the joint probability for finding a tube segment at the position $s$ and with the orientation $\mathbf{u}$. We choose the normalization condition $\int_{0}^{1} \int \psi d^{3} u d s=1$, independent of position $\mathbf{r}$ and time $t$, for the probability density $\psi$ with $\psi \geq 0$.

In the original DE model, chain stretching was neglected. It has long been recognized that chain stretching is important at high shear rates (comparable to the reciprocal of the Rouse relaxation time of the molecule), in extensional flows, and for understanding very rapid double step-strain deformations. With the notation of Eqn. (1) let $\lambda=\sqrt{\operatorname{tr} \mathbf{A}}$ denote the ratio between the contour length of the primitive chain and its length at equilibrium. Instead of incorporating $\lambda$ into the list of arguments of the configurational distribution function and considering the contour length fluctuation effect, it is kept as a separate and deterministic variable in the SU model (see [34,10] for the reasons). 
After determining all the state variables, the model has been formulated within the GENERIC framework by using its "building blocks" step by step, as for the SU model $[34,10]$. In the following, we summarize the final time-evolution equations for the structural variables and draw attention to the modifications brought to bear on the original SU model.

The equation for the chain contour length stretching and relaxation reads

$$
\frac{D \lambda}{D t}=\dot{\lambda}=\dot{\lambda}_{\text {convect }}+\dot{\lambda}_{\text {dissip }}
$$

where $D / D t$ is the material time derivative, and the total stretching rate is split into convective and dissipative contributions,

$$
\dot{\lambda}_{\text {convect }}=\lambda \boldsymbol{\kappa}: \mathbf{S},
$$

and

$$
\dot{\lambda}_{\text {dissip }}=-\frac{1}{\tau_{s}} \frac{c(\lambda)}{3 Z}(\lambda-1) .
$$

In Eq. (7), $\boldsymbol{\kappa}$ is the transpose of the velocity gradient tensor and $\mathbf{S}$ is the symmetric second moment orientation tensor defined by

$$
\mathbf{S}=\int_{0}^{1} \int \mathbf{u u} \psi(\mathbf{u}, s, \mathbf{r}, t) d^{3} u d s
$$

In Eq. (8), $Z$ is the number of entanglement segments per chain, $\tau_{s}$ is the characteristic stretching time, and $c(\lambda)$ is an effective spring coefficient $[11,10]$

$$
c(\lambda)=\frac{3 Z \lambda_{\max }^{2}(\lambda+1)}{\lambda\left(\lambda_{\max }^{2}-\lambda^{2}\right)} .
$$

The parameter $\lambda_{\max }$ here is the maximum possible stretching ratio of the chain contour length.

The Fokker-Planck (FP) equation for the configurational distribution function takes the form

$$
\begin{aligned}
\frac{\partial \psi}{\partial t}= & -\frac{\partial}{\partial \mathbf{u}} \cdot\left[\left(\mathbf{1}-\frac{\mathbf{u u}}{|\mathbf{u}|^{2}}\right) \cdot \boldsymbol{\kappa} \cdot \mathbf{u} \psi\right]-\frac{\partial}{\partial s}\left(\dot{s}_{t o t} \psi\right) \\
& -\frac{\dot{\lambda}_{\text {dissip }}}{\lambda} \psi+\frac{1}{\pi^{2} \tau_{d}} \frac{\partial^{2} \psi}{\partial s^{2}}+\frac{\partial}{\partial \mathbf{u}} \cdot D\left(\mathbf{1}-\frac{\mathbf{u u}}{|\mathbf{u}|^{2}}\right) \cdot \frac{\partial}{\partial \mathbf{u}} \psi
\end{aligned}
$$

where the drift velocity

$$
\dot{s}_{\text {tot }}=-\frac{1}{\lambda}\left(s-\frac{1}{2}\right) \dot{\lambda}_{\text {dissip }}
$$

and $D$ is an orientational diffusivity coefficient (see [11] or [23] for more details). The drift velocity for $s$ means that there is a rescaling of this position 
label for the tube segment only when the chain contour length relaxes in the tube. The first term on the right-hand side of Eq. (11) represents the standard corotational behavior of the unit orientation vector $\mathbf{u}$, the third term (creation/destruction term) compensates for configurations lost or gained at the boundaries. The terms involving second-order derivatives in Eq. (11) are of irreversible nature and express the erratic reptational motion along the chain contour (second-order derivative with respect to $s$ with reptation time $\tau_{d}$ ) and constraint releases (second-order derivative with respect to $\mathbf{u}$ with the diffusion coefficient $D$ ) respectively.

So far, the model presented is formally the same as the SU model. Next, we will introduce two modifications.

The modified expression for $D$ is $D=1 /\left(6 \tau_{\text {eff }}\right)$ where

$$
\tau_{e f f}=\max \left(\frac{1}{\frac{1}{\tau_{d}}+\beta_{1} k H(k)}, \tau_{R}\right),
$$

$H=H(k)$ is the Heaviside step function and

$$
k=\boldsymbol{\kappa}: \mathbf{S}-\frac{\dot{\lambda}}{\lambda} .
$$

The diffusion coefficient $D$, defined using Eq. (13), accounts for double reptation (through $\tau_{d}$ ) and CCR (through $k$ ), as previously explained in the SU model [11]. The factor $\beta_{1}$ appearing in Eq. (13) allows CCR2 to be represented in the same manner as for CCR and $\beta_{1} k H(k)$ determines the effective orientation relaxation rate caused by constraint releases. We note that in addition to the double reptation and CCR terms in (13) there is a third term proportional to the Rouse time $\tau_{R}$. Without such a term the orientational relaxation time would tend to zero in the limit of very fast flows. The additional term is an irreducible friction term expressing the fact that once the topological contribution to the chain friction is swept away the orientational relaxation time of the now unentangled chain drops to the Rouse time $\tau_{R}$ (see the papers of Ianniruberto and Marrucci $[19,20])$. Eq. (13) describes a monotonic variation of $D$ from $1 /\left(6 \tau_{d}\right)$ to $1 /\left(6 \tau_{R}\right)$ due to CCR and the argument just mentioned.

In opposition to the point of view expressed by some in the literature [19,20], we here argue that a similar physical mechanism also applies to chain length relaxation. That is, $\tau_{s}$ should also vary with CCR from the value at equilibrium under constraint to the value corresponding to a somehow unconstrained Rouse chain, because of the fast removal of constraints. Hence we propose

$$
\tau_{s}=\max \left(\frac{1}{\frac{1}{\tau_{R 0}}+\beta_{2} k H(k)}, \tau_{R}\right)
$$


where $\tau_{R 0}$ is the primary Rouse time at equilibrium and the factor $\beta_{2}$ has the same role as $\beta_{1}$, i.e. $\beta_{2} k H(k)$ is the effective stretching relaxation rate caused by constraint releases. We choose $\tau_{d} / \tau_{R}=3 Z$ and $\tau_{d} / \tau_{R 0}=Z$ consistent with the fact that at equilibrium (entangled case) stretch relaxation is simply along the contour path of the chain and thus essentially one-dimensional, whereas the assumption underlying the choice of the pre-factor 3 in the original Doi-Edwards choice of $3 Z$ for the reptation to Rouse time ratio was that relaxation occurred in three dimensions [41]. Stretch relaxation therefore takes place over a longer time under equilibrium conditions than in a fast flow, as seems intuitively reasonable. Note that it has been usual in the literature (see, for example, [11]) to choose a fixed ratio $\tau_{d} / \tau_{s}=3 Z$ for the characteristic reptation and stretching times.

The elastic stress $\boldsymbol{\tau}$ is related to the orientation tensor $\mathbf{S}$ in both the $\mathrm{SU}$ and modified SU models by

$$
\boldsymbol{\tau}=5 G_{N}^{0}\left[1-\frac{\lambda^{2}-1}{1-\left(\lambda / \lambda_{\max }\right)^{2}}\right] \mathbf{S},
$$

where $G_{N}^{0}=\frac{3}{5} \nu_{2} k_{B} T$ is the plateau modulus and $\nu_{2}$ is the segment density. The modified SU model presented above accounts for reptation, double reptation, CCR, CCR2, Rouse friction and chain stretching, whilst avoiding IAA. The boundary conditions for the FP equation (11) and the expression for the extra stress tensor (16) remain the same as for the SU model. The model is thermodynamically admissible in the sense that it possesses the full structure of GENERIC and satisfies all the required properties for the building blocks therein [10]. The model has only four structural degrees of freedom, one from the position labels $s$, two from the unit orientation vector $\mathbf{u}$, and one from the chain stretching $\lambda$. In addition to the plateau modulus $G_{N}^{0}$ and the reptation time $\tau_{d}$, the model has only four parameters $\left(Z, \lambda_{\max }, \beta_{1}, \beta_{2}\right)$. We regard $G_{N}^{0}$ and $\tau_{d}$ as the key adjustable parameters, $\beta_{1}$ and $\beta_{2}$ are parameters of order unity, while $Z$ and $\lambda_{\max }$ are fixed by the chemistry of a particular polymer.

\subsection{Modified encapsulated FENE dumbbell (EFD) model}

In the derivation of the FP equation for the classical FENE dumbbell model, appropriate only for modelling dilute polymer solutions, the hydrodynamic drag force $\mathbf{F}_{i}^{h}$ (say) on bead $i(i=1,2)$ is taken to be directly proportional to the difference between the velocity-space averaged bead velocity $\ll \dot{\mathbf{r}}_{i} \gg$ and the solvent velocity $\mathbf{v}\left(\mathbf{r}_{i}\right)$, where $\mathbf{r}_{i}$ denotes the position vector of bead $i$. That is, we write the drag force as

$$
\mathbf{F}_{i}^{h}=\boldsymbol{\zeta}\left(\ll \dot{\mathbf{r}}_{i} \gg-\mathbf{v}\left(\mathbf{r}_{i}\right)\right),
$$


where $\boldsymbol{\zeta}=\zeta \boldsymbol{\delta}$ is an isotropic friction tensor and $\zeta$ is the so-called friction coefficient.

In a concentrated polymer solution or melt the motion of a molecule is restricted by the presence of other molecules and it is appropriate under these circumstances that the hydrodynamic drag should be anisotropic. Let $\mathbf{Q}$ be the dumbbell end-to-end vector and $\mathbf{u}=\mathrm{Q} / Q$ a unit vector in the direction of Q. Then the Fokker-Planck equation for the original EFD model [4] without considering the anisotropy of Brownian motion is

$$
\frac{\partial \psi}{\partial t}=-\frac{\partial}{\partial \mathbf{Q}} \cdot\left[\boldsymbol{\kappa} \cdot \mathbf{Q} \psi-2 \zeta^{-1} \cdot \mathbf{F}^{(c)} \psi-2 k_{B} T \boldsymbol{\zeta}^{-1} \cdot \frac{\partial \psi}{\partial \mathbf{Q}}\right]
$$

where $\boldsymbol{\zeta}$ is now an anisotropic friction tensor given by

$$
\zeta=\zeta\left(\mathbf{u u}+\sigma^{-1}(\boldsymbol{\delta}-\mathbf{u u})\right)
$$

having inverse

$$
\boldsymbol{\zeta}^{-1}=\zeta^{-1}(\mathbf{u u}+\sigma(\boldsymbol{\delta}-\mathbf{u u}))
$$

and

$$
\mathbf{F}^{(c)}=\frac{H \mathbf{Q}}{1-Q^{2} / Q_{\max }^{2}},
$$

is the usual FENE spring law. We note that when the parameter $\sigma=1$ the friction tensor is isotropic and that when $\sigma<1$ the molecule experiences greater resistance to movement in a direction normal to the connector vector Q.

We now introduce a dimensionless end-to-end vector

$$
\mathrm{Q}^{*}=\mathrm{Q} / \sqrt{\frac{k_{B} T}{H}}
$$

and write the Fokker-Planck equation (18) in dimensionless form (dropping the asterisks) as

$$
\frac{\partial \psi}{\partial t}=-\frac{\partial}{\partial \mathbf{Q}} \cdot\left[\boldsymbol{\kappa} \cdot \mathbf{Q} \psi-\frac{1}{2 \lambda_{H}} \mathbf{F}^{(c)} \psi-\frac{1}{2 \lambda_{H}}[\mathbf{u u}+\sigma(\boldsymbol{\delta}-\mathbf{u u})] \cdot \frac{\partial \psi}{\partial \mathbf{Q}}\right],
$$

where $\lambda_{H}=\zeta /(4 H)$ is a characteristic relaxation time and the non-dimensional force law appearing in (23) is given by

$$
\mathbf{F}^{(c)}=\mathbf{Q} /\left(1-\frac{Q^{2}}{b}\right)
$$

where the dimensionless maximum spring extensibility $b$ is defined by

$$
b=Q_{\max }^{2}
$$


Let us introduce $\tau_{s}=2 \lambda_{H}$ and $D=\sigma /\left(2 \lambda_{H}\right)$. Then the Itô stochastic differential equation corresponding to (23) (see [32], for example) is

$$
d \mathbf{Q}=\left\{\boldsymbol{\kappa} \cdot \mathbf{Q}-\frac{1}{\tau_{s}} \mathbf{F}^{(c)}+\left(\frac{2}{\tau_{s}}-2 D\right) \frac{\mathbf{u}}{Q}\right\} d t+\left[\sqrt{\frac{2}{\tau_{s}}} \mathbf{u u}+\sqrt{2 D}(\boldsymbol{\delta}-\mathbf{u u})\right] \cdot d \mathbf{W} .
$$

The new encapsulated dumbbell model that we propose in this paper involves a modification to the two parameters $\tau_{s}$ and $D$ that appear in Eqn. (26) and a change of significance for the vector $\mathbf{u}$ which henceforth represents a unit vector tangential to a segment backbone in a chain of $Z$ (say) segments. We write $D=1 /\left(6 \tau_{\text {eff }}\right)$ where the expression that we use for $\tau_{\text {eff }}$ is

$$
\tau_{\text {eff }}=\max \left(\frac{1}{\frac{2}{\tau_{d}}+\beta_{1} k H(k)}, \tau_{R}\right),
$$

where $H=H(k)$ is the Heaviside step function with

$$
k=\boldsymbol{\kappa}:\langle\mathbf{u u}\rangle-\frac{\langle\dot{Q}\rangle}{\langle Q\rangle}
$$

The expression (27) for $\tau_{\text {eff }}$ bears a strong resemblance to that in (13). However, we note that in the modified SU model (as in the original SU model) reptation of the probe chain is modelled by the dynamics of the variable $s$, whereas in the modified EFD and the coupled DCR-CS models both reptation and double reptation are modelled directly through the effective orientation relaxation time $\tau_{\text {eff }}$. The consequence of the different modelling of reptation in the three models is that a term $2 / \tau_{d}$ is required in (3) and (27) but only a term $1 / \tau_{d}$ in $(13)$.

We note in (27) that $\tau_{\text {eff }}$ is independent of $k$ for $k<0$. This is because negative $k$ means that the tube-chain relative motion is inverted and we consider that the tube is not destroyed by this expansion-like motion. When the tube-chain relative motion is inverted as just described, chains are expelled at the ends of their tubes and are thus free to renew their orientation. In the original and modified SU models (see Sec. 2.2), this orientation renewal is automatically included through the drift and creation/destruction terms in Eqn. (11) together with the boundary conditions at the chain ends. If we wanted to model this effect through the effective relaxation time in (27) we could simply use the absolute value $|k|$ instead of $k H(k)$, similar to what is done in the decoupled DCR-CS model of Ianniruberto and Marrucci [19]. However, even with this modification in place only a small quantitative difference in the shear stress for the reversing double-step strain flow experiment described in Sec. 3.4 , could be observed.

We here argue that $\tau_{s}$ should also vary with CCR from the value at equilib- 
rium under constraint to the value corresponding to a somehow unconstrained Rouse chain, because of the fast removal of constraints. Hence we propose

$$
\tau_{s}=\max \left(\frac{1}{\frac{1}{\tau_{R 0}}+\beta_{2} k H(k)}, \tau_{R}\right)
$$

where $\tau_{R 0}$ is the primary Rouse time at equilibrium, and as explained in Section 2.2, we choose $\tau_{d} / \tau_{R}=3 Z$ and $\tau_{d} / \tau_{R 0}=Z$ consistent with the fact that at equilibrium (entangled case) stretch relaxation is simply along the contour path of the chain and thus essentially one-dimensional, whereas the assumption underlying the choice of the pre-factor 3 in the original Doi-Edwards choice of $3 Z$ for the reptation to Rouse time ratio was that relaxation occurred in three dimensions [41].

Finally, the polymeric stress is given by

$$
\tau=G\left\langle\frac{\mathrm{QQ}}{1-Q^{2} / b}\right\rangle
$$

where $G=\nu_{3} k_{B} T$ is an elastic modulus and $\nu_{3}$ denotes the segment density.

\subsection{Choice of equivalent parameters for the coupled DCR-CS, modified SU} and modified EFD models

The parameters $\tau_{d}, G$ and $G_{N}^{0}$ appearing in Eqns. (3), (5), (13), (16), (27) and (30) are chosen in such a way that the cross-over point of the respective steady shear stress and first normal stress difference curves is predicted to be equal to that from the experimental data. As will be noted in the next section, this procedure leads to broadly similar choices of $\tau_{d}$ when two models are being used to simulate the same fluid, although the corresponding moduli $G$ and $G_{N}^{0}$ may differ greatly.

Two parameters require a careful choice to ensure equivalence in the different models when the same fluid is being simulated: the Rouse time $\tau_{R}$ (see Eqns. (3), (13) and (27)) and the non-dimensional maximum finite extensibility parameter $b$ (see Eqns. (4), (10)(where $b=\lambda_{\text {max }}^{2}$ ) and (25)). A fair comparison of results between the modified EFD model and the coupled DCR-CS model requires that the Rouse time for the latter be chosen equal to a half of the value that would be taken for the decoupled DCR-CS model: see Wapperom and Keunings [43] for a discussion of this point. In the present context, this also means that we should choose $\tau_{R}$ for the coupled DCR-CS model to be a half of that used for the modified EFD model when a comparison of results is being performed. 
The extensibility parameter $b$ in the three models is not the same due to the different definitions used for the maximum stretch ratio of a chain segment. The relationship between the $b\left(=b_{D C R}\right.$, say) parameter used in the coupled DCR-CS model and that of the modified EFD model $\left(b=b_{E F D}\right.$, say) is

$$
b_{D C R}=b_{E F D} \times \frac{\left(\frac{k_{B} T}{H}\right)}{a^{2}},
$$

where $a$ is the equilibrium length of a chain segment in the EFD model. Conveniently, it may be shown that the equilibrium probability density function $\psi_{e q}(\mathbf{Q})$ for the EFD models (original and modified) is the same as that for the standard FENE model and is thus given

$$
\psi_{e q}=C\left(1-\frac{Q^{2}}{b_{E F D}}\right)^{b_{E F D} / 2}
$$

where $C$ is a normalizing constant and the notation in (32) is the same as in Sec. 2.3. From the relation (31) it may be shown after a few manipulations and using spherical polar coordinates that

$$
\begin{aligned}
b_{E F D} & =b_{D C R} \times \frac{\int_{r=0}^{\sqrt{b_{E F D}}} r^{4}\left(1-r^{2} / b_{E F D}\right)^{b_{E F D} / 2} d r}{\int_{r=0}^{\sqrt{b_{E F D}}} r^{2}\left(1-r^{2} / b_{E F D}\right)^{b_{E F D} / 2} d r} \\
& =b_{D C R} \times b_{E F D} \times \frac{\int_{u=0}^{1} u^{3 / 2}(1-u)^{b_{E F D} / 2}}{\int_{u=0}^{1} u^{1 / 2}(1-u)^{b_{E F D} / 2}}, \\
& =b_{D C R} \times b_{E F D} \times \frac{B\left(\frac{5}{2}, 1+\frac{b_{E F D}}{2}\right)}{B\left(\frac{3}{2}, 1+\frac{b_{E F D}}{2}\right)} \\
\Rightarrow b_{E F D} & =3 b_{D C R}-5,
\end{aligned}
$$

where, in $(33) B(m, n)=\frac{\Gamma(m) \Gamma(n)}{\Gamma(m+n)}(m>0, n>0)$ denotes the Beta function. We note that $\lambda_{\text {max }}^{2}\left(\mathrm{SU}\right.$ model) is the same as $b_{D C R}$.

\section{$3 \quad$ Numerical results}

For the results presented in Fig. 1 we used a Fokker-Planck-based high-order numerical method, the details of which may be found in [23]. For all other results in this Section a Brownian dynamics simulation was used with between $10^{4}$ and $10^{6}$ configurations and time step sizes $\Delta t$ chosen so that the strain in all flows considered per time step never exceeded 0.02 . For small deformation rates the maximum time step size was set at $4 \times 10^{-4}$. Further details of our Brownian dynamics method may be gleaned from [11]. 
The results of comparing the predictions of the SU model and the modified SU model with experimental data for steady simple shear flow are presented in Fig. 1. In this figure the steady-state values of the shear stress $\tau_{x y}$ and the first normal stress difference $N_{1}$ are plotted as functions of the shear rate $\dot{\gamma}$. The experimental data used for comparison are taken from the thesis of Kahvand [21]. The test fluid is a solution in tricresyl phosphate of nearly monodisperse polystyrene with a molecular weight $M_{w}$ of $1.9 \times 10^{6}$ (polydispersity index of 1.2 ) and a polymer density of $0.135 \mathrm{~g} / \mathrm{cm}^{3}$. The average number of entanglements $(Z)$ of the fluid is estimated to be 10 . In [11] $Z$ was taken equal to 7 with 441 Kuhn steps per entanglement. Hence, we here estimate the number of Kuhn steps per entanglement to be $7 \times 441 / 10=308.7$. The maximum chain stretching ratio $\left(\lambda_{\max }\right)$ of the fluid is equal to the square root of the number of Kuhn steps per entanglement according to the theory in [11] and is thus estimated in the present paper to be 17.6.

As explained in Sec. 2.4 the parameters $\tau_{d}, G_{N}^{0}$ and $G$ in the SU and EFD models are chosen in such a way that the cross-over point of the steady shear stress and first normal stress difference curves is predicted correctly. For the two SU models, we then obtain $\tau_{d}=15 \mathrm{~s}$ and $G_{N}^{0}=1160 \mathrm{~Pa}$. With a value of $\lambda_{\max }=17.6$ both the predictions of the original SU model and those of the modified SU model leave something to be desired. Firstly, the SU model predicts a slight decrease in the shear stress over a range of shear rates that extends from roughly $\dot{\gamma} \approx 0.2 s^{-1}$ to $\dot{\gamma} \approx 2 s^{-1}$, which is inconsistent with the data and indicates an instability. Then, although good for low shear rates, the modified SU model predicts a sharp increase in the shear stress and first normal stress difference for $\dot{\gamma}>10 \mathrm{~s}^{-1}$, way above what has been measured experimentally. This increase corresponds to unrealistic shear thickening, similar to what has been observed with the decoupled DCR-CS model [19] by Wapperom et al. [42]. However, we found that a much smaller value (1.75) of $\lambda_{\max }$ gives more realistic predictions at high shear rates. The predictions of the shear stress by the modified SU model with the smaller value of $\lambda_{\max }$ are now in much better agreement with the experimental data over the whole range of shear rates and the first normal stress difference increases gradually with shear rate, consistent with the experimental data. We note in passing that a similar reduction in $\lambda_{\max }$ brought about only a marginal improvement in the predictions of the original SU model. The same need for $\lambda_{\max }$ smaller than the theoretically determined value was documented by Ianniruberto and Marrucci [19] in their comparisons of shear data with the experimental results of Bercea et al. [1] for a semi-dilute solution of PMMA in toluene.

For all the fact that we have registered an improvement in the predictive capacities of the modified SU model by taking $\lambda_{\max }=1.75$, this situation is far 


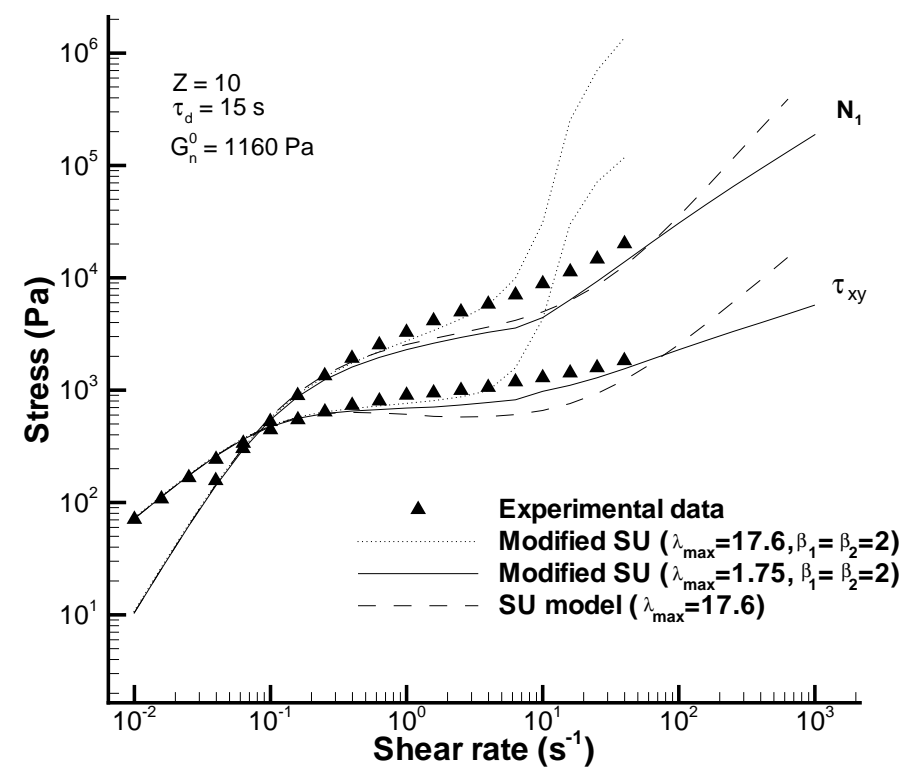

Fig. 1. Steady-state values of shear stress $\tau_{x y}$ and first normal stress difference $N_{1}$ as functions of shear rate $\dot{\gamma}$. Shown are the predictions of the SU model, the modified SU model and experimental data [21].

from ideal. Numerical evidence that the need for an unphysically small value of $\lambda_{\max }$ finds its origin in the decoupling of Eqn. (6) for the chain stretch $\lambda$ from Eqn. (11) is presented in Fig. 2. Here, with the fully coupled modified EFD model a much larger stretching of the chain contour length $\left(b=3 \lambda_{\max }^{2}-5 \approx\right.$ 924 ) is possible whilst faithfully predicting the viscometric functions over the entire range of shear rates.

\subsection{Modified EFD model: start-up shear flow}

In Figs. 3 and 4 we examine the predicted growth of the normalized shear stress and first normal stress difference for start-up shear flow. The experimental data (denoted by symbols in the two figures) are for the same fluid as in Section 3.1 and the results are taken from the thesis of Kahvand [21]. The modified EFD model parameters remain the same as in the previous section. The modified EFD model overpredicts the $\tau_{x y}$ overshoot (see Fig. 3) at the highest shear rate $\dot{\gamma}=10 \mathrm{~s}^{-1}$ and underpredicts that of $N_{1}$ (see Fig. 4). Reference should be made to Figs. 2-4 of [11] for a comparison of the performance of the modified EFD model against that of the SU model [34], the model of Hua et al. [15] and the model of Mead et al. [28]. We note that both the models of Hua et al. [15] and Mead et al. [28] also overpredict the $\tau_{x y}$ overshoot at $\dot{\gamma}=10 \mathrm{~s}^{-1}$ and that the modified EFD model does a better job at approximating the $N_{1}$ 


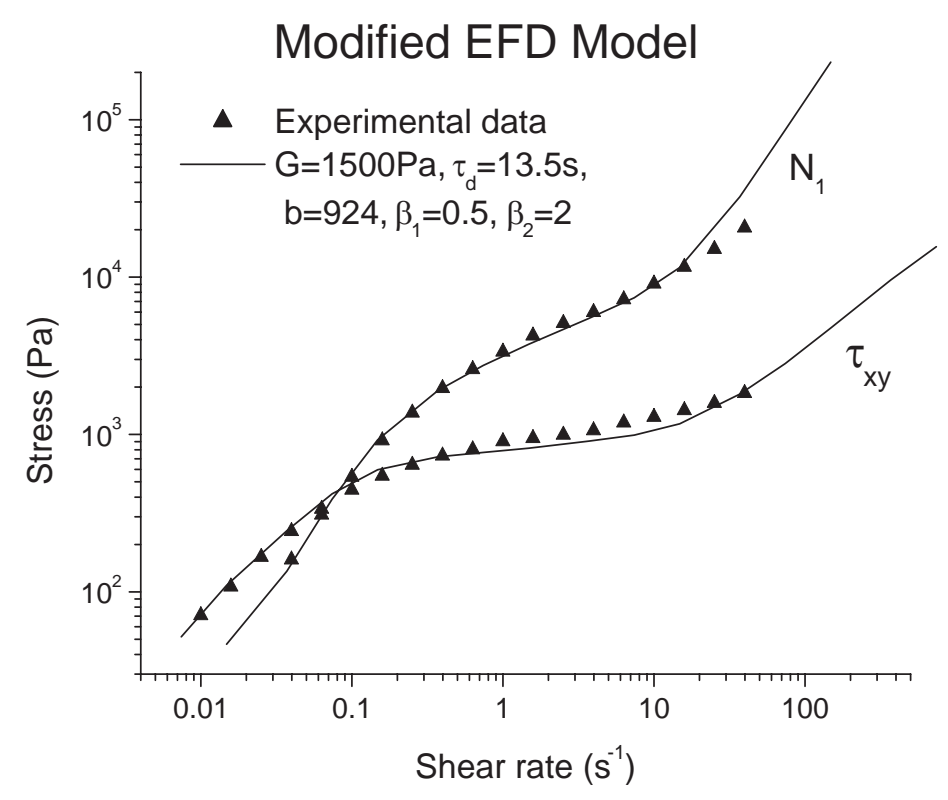

Fig. 2. Steady-state values of shear stress $\tau_{x y}$ and first normal stress difference $N_{1}$ as functions of shear rate $\dot{\gamma}$. Shown are the predictions of the modified EFD model and experimental data [21].

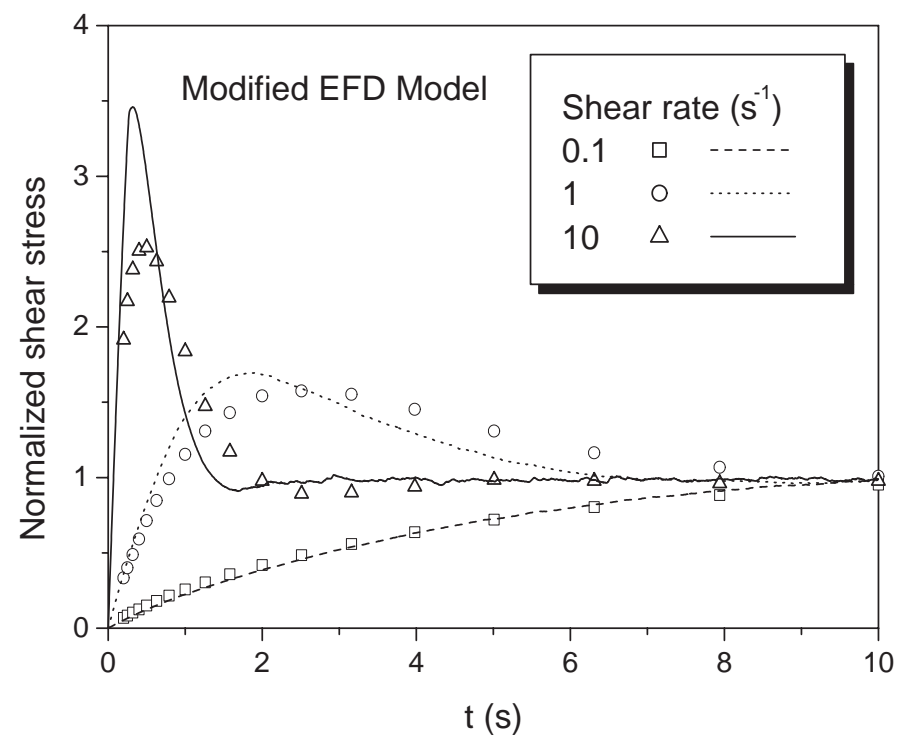

Fig. 3. Transient growth of normalized shear stress as a function of time under start-up shear flow at several shear rates predicted by the modified EFD model (curves) and experiments [21] (symbols).

overshoot at this shear rate than the SU model. We see from Fig. 4 that an overshoot in $\tau_{x y}$ occurs even at $\dot{\gamma}=1$ and thus we concur with the observation made in [11] that an overshoot in $N_{1}$ occurs at a higher shear rate than that in $\tau_{x y}$. At $\dot{\gamma}=10 s^{-1}$ the peak value of $N_{1}$ is attained later than that of $\tau_{x y}$. 


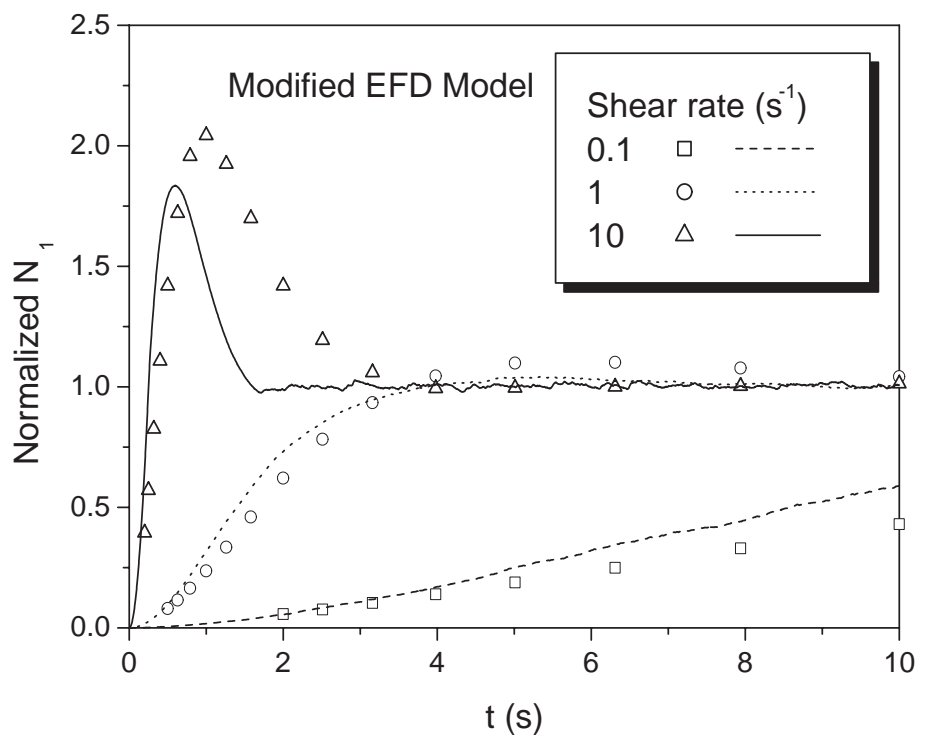

Fig. 4. Transient growth of first normal stress difference as a function of time under start-up shear flow at several shear rates predicted by the modified EFD model (curves) and experiments [21] (symbols).

3.3 EFD model, coupled DCR-CS model and modified EFD model: steady shear flow and steady uniaxial extensional flow

In this subsection we discuss the extent of agreement with experimental data of predictions of the coupled DCR-CS model and the modified EFD model for steady simple shear flow and steady uniaxial extensional flow. In both flows the fluid data comes from experimental measurements by Bhattacharjee et al. [2,3] for a $10 \%$ solution of $3.9 \times 10^{6}$ molecular weight polystyrene in diethyl phthalate. The number of entanglements in the solution was calculated to be $Z=27.4$ and the maximum stretching ratio of the chain contour length to be $\lambda_{\max }=13.6$. The Rouse time was given by the authors as $\tau_{R}=0.282 \mathrm{~s}$ and therefore $\tau_{d}=3 Z \tau_{R}=23.18 s$.

As with the results presented in Section 3.1 the reptation time $\tau_{d}$ and elastic modulus $G$ used for the simulations shown in Figs. 5-10 were calculated to give the correct crossover point for the shear stress and first normal stress difference. We observe that although the predicted reptation times for the coupled DCR-CS model $(17 s)$ and the modified EFD model $(18 s)$ are in reasonable agreement, there is a factor of 2 difference between their respective elastic moduli. For the modified EFD model and the coupled DCR-CS model $Z$ was taken equal to 20. Thus, in order to preserve the same total number of Kuhn steps as given by Bhattacharjee et al. [2,3] the new number of Kuhn steps per entanglement is calculated to be $27.4 \times(13.6)^{2} / 20 \approx 253$. In the coupled DCR-CS model $b=\lambda_{\max }^{2}$ and is therefore chosen equal to 253. For the EFD model and modified EFD model, as explained in Sec. 2.4, $b$ is taken to be 
$3 \times 253-5=754$. With a value of $\tau_{d}=17 \mathrm{~s}$ for the coupled DCR-CS model, the correct $\tau_{R}$ (see discussion in Sec. 2.4$)$ is $(1 / 2) \times \tau_{d} / 3 Z=17 / 120=0.142 s$. The Rouse time for the modified EFD model is $18 / 60=0.3 \mathrm{~s}$, thus close to the estimated value of Bhattacharjee et al.

For the shear data the coupled DCR-CS model (Fig. 5) would seem to do a reasonable job in predicting the shear stress. However, the model noticeably underpredicts the first normal stress difference $N_{1}$ in the shear-rate range $\dot{\gamma} \approx 0.1 s^{-1}$ to $\dot{\gamma} \approx 10 s^{-1}$. By a careful selection of the friction anisotropy parameter $\sigma$ the EFD model is able to furnish convincing quantitative agreement with either the shear stress $(\sigma=0.02)$ or first normal stress difference $(\sigma=0.01)$ data (see Fig. 6). However, no single choice of $\sigma$ will do for both. In contrast (Fig. 7), the modified EFD model gives excellent agreement with the experimental shear flow results with a single choice of parameter set. In Fig. 7 we have plotted three curves for both $N_{1}$ and $\tau_{x y}$. The dashed and dotted curves in both cases have been generated with the simple choice $\tau_{s}=\tau_{R}$ with two different values for $\beta_{1}$ (as indicated in the figure legend), in order to investigate the effect on the predictive capabilities of the modified EFD model of allowing $\tau_{s}$ to vary (or not) with CCR. Although the choice (29) for $\tau_{s}$ gives superior results to those of simply choosing $\tau_{s}=\tau_{R}$, the improvement is, admittedly, only modest.

Despite the good agreement for the shear data the coupled DCR-CS model fails to adequately match the uniaxial extensional data of Bhattacharjee et al. for the same choice of model parameters. In Fig. 8 it may be seen that the model significantly under-predicts the experimental data over the range $\dot{\epsilon} \approx 0.1 s^{-1}$ to $\dot{\epsilon} \approx 3 s^{-1}$. Bhattacharjee et al. [3] have already noted the poor performance of the Mead-Larson-Doi model [28] for this class of flows and surmised that the failure may have been due to the non-inclusion of diffusive constraint release and the corresponding dual constraint release version of the CCR mechanism. The single-mode decoupled DCR-CS model of Ianniruberto and Marrucci [19] did rather better for extensional flow, despite the shear-thickening behaviour at high shear rates for steady shear flow, noted previously. The present performance of the coupled DCR-CS model in uniaxial extensional flow is therefore rather disappointing. From Fig. 9 it is clear that the EFD model is unsatisfactory in its predictions for extension rates $\dot{\epsilon}>1 s^{-1}$. The modified EFD model represents an improvement over the EFD model and its stress difference curves in Fig. 10 are certainly closer to the experimental data for extension rates $\dot{\epsilon}<3 s^{-1}$ than either of the other models. The dotted curve in Fig. 10 was plotted by taking $\beta_{1}=1$ in Eqn. (27) and $\tau_{s}=\tau_{R}$. The improvement registered by incorporating CCR into $\tau_{s}$ is noticeable but small. 


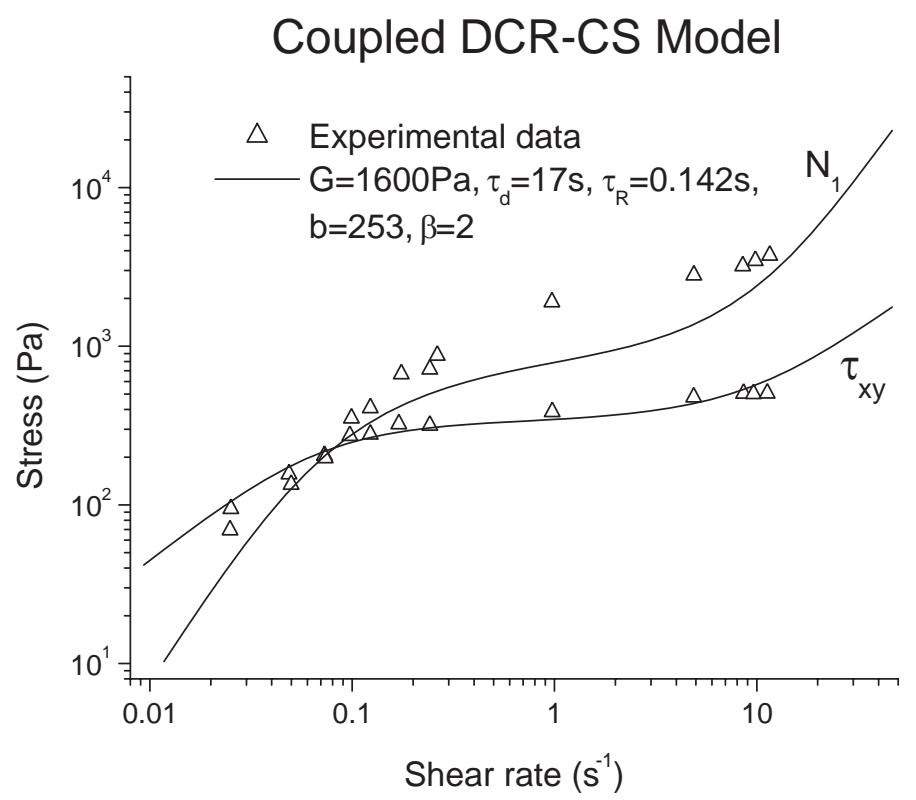

Fig. 5. Steady-state values of shear stress $\tau_{x y}$ and first normal stress difference $N_{1}$ as functions of shear rate $\dot{\gamma}$. Shown are the predictions of the coupled DCR-CS model and experimental data [2].

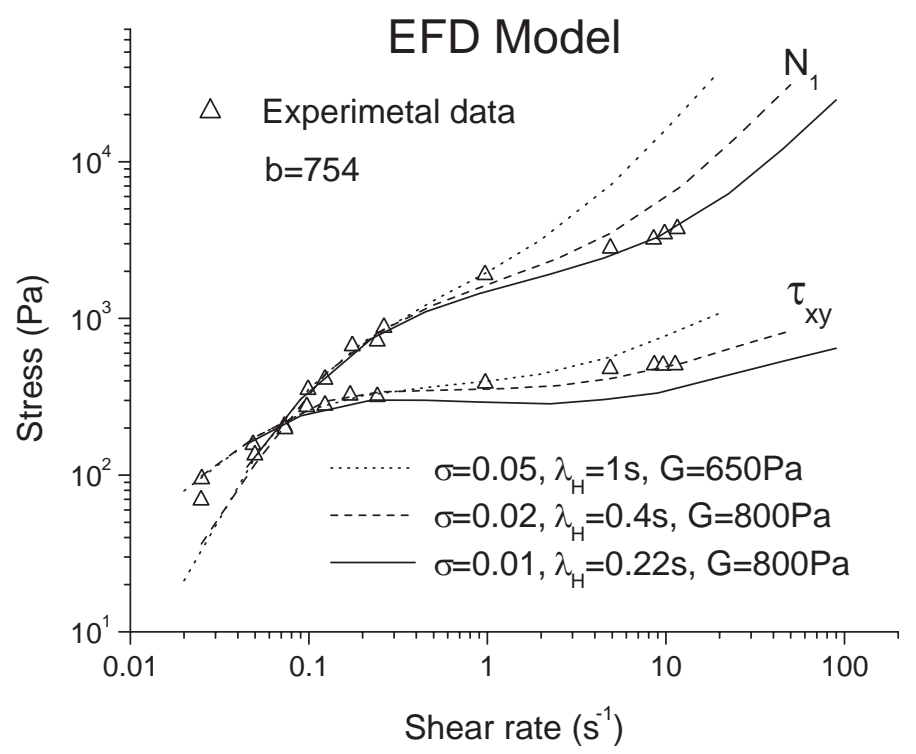

Fig. 6. Steady-state values of shear stress $\tau_{x y}$ and first normal stress difference $N_{1}$ as functions of shear rate $\dot{\gamma}$. Shown are the predictions of the EFD model for different values of the anisotropy parameter $\sigma$ and experimental data [2].

\subsection{Modified EFD model and coupled DCR-CS model: reversing double-step strain flow}

In a double-step shear strain experiment a strain of $S_{1}$ is imposed and then at a time $t_{w}$ (say) later a strain of $S_{2}$ is imposed, giving a total strain of $S_{1}+S_{2}$. 


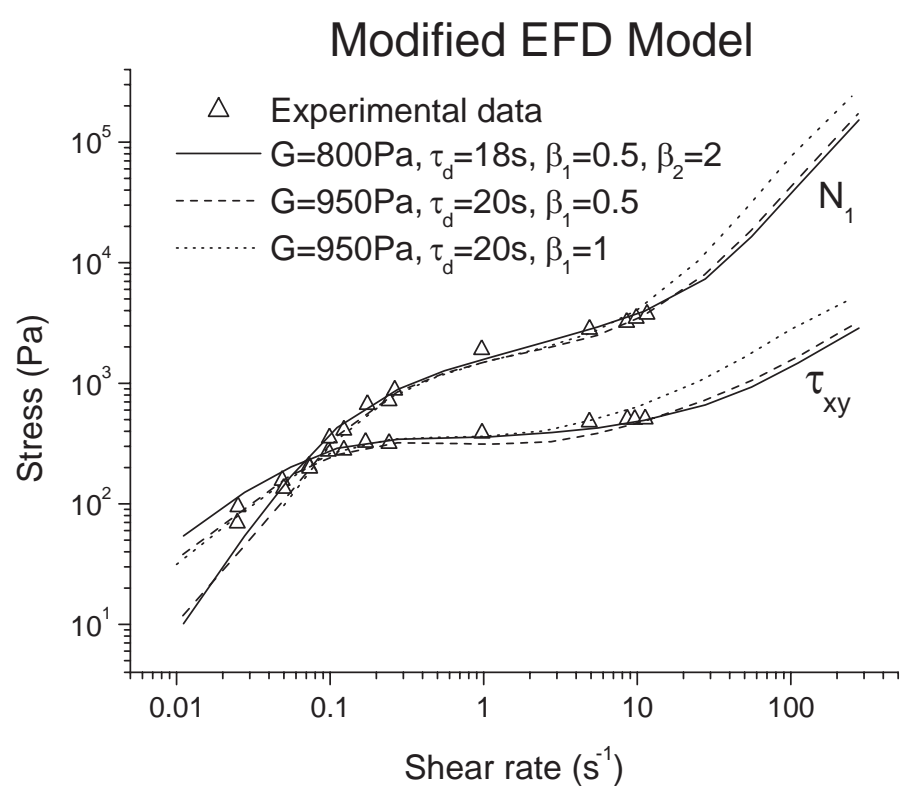

Fig. 7. Steady-state values of shear stress $\tau_{x y}$ and first normal stress difference $N_{1}$ as functions of shear rate $\dot{\gamma}$. Shown are the predictions of the modified EFD model and experimental data [2]. The dashed and dotted curves were generated with the choice of $\beta_{1}$ as shown and $\tau_{s}=\tau_{R}$.

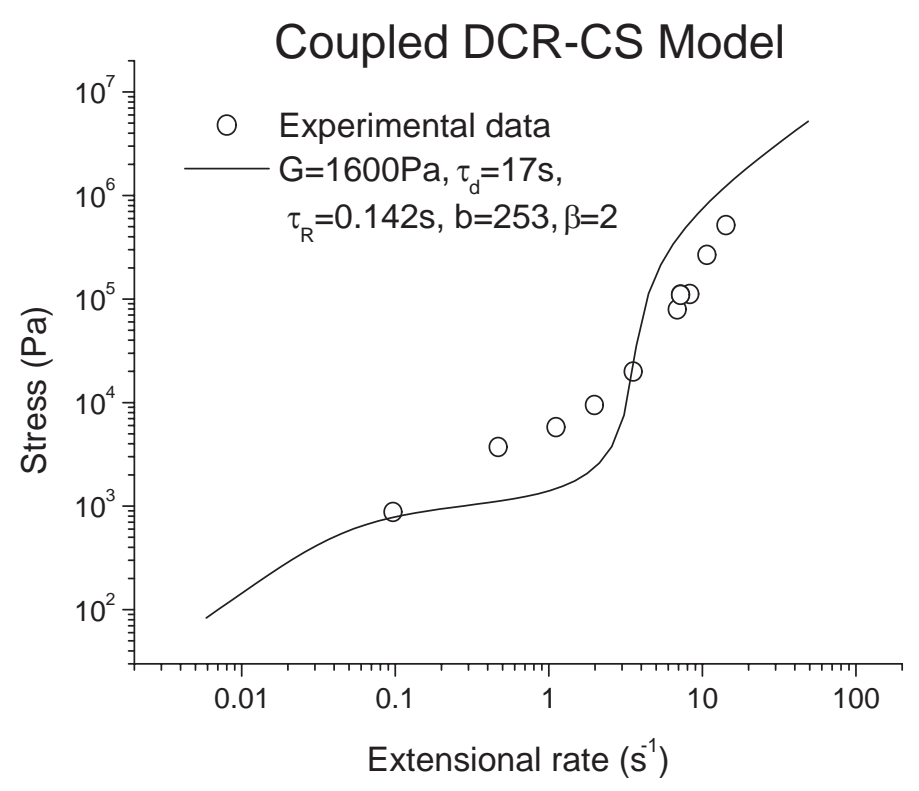

Fig. 8. Steady-state values of the first normal stress difference $\tau_{x x}-\tau_{y y}$ in uniaxial extensional flow. Shown are the predictions of the coupled DCR-CS model and experimental data [3].

In Figs. 11 and 12 we show the results for the normalized shear stress and for different values of $t_{w} / \tau_{d}$ of a reversing double-step strain, where $S_{1}=4$ and $S_{2}=-2$. The model parameters are the same as those of the previous subsection. Although we do not quantitatively compare the coupled DCR-CS model and modified EFD model results with those from experiments (to do so, 


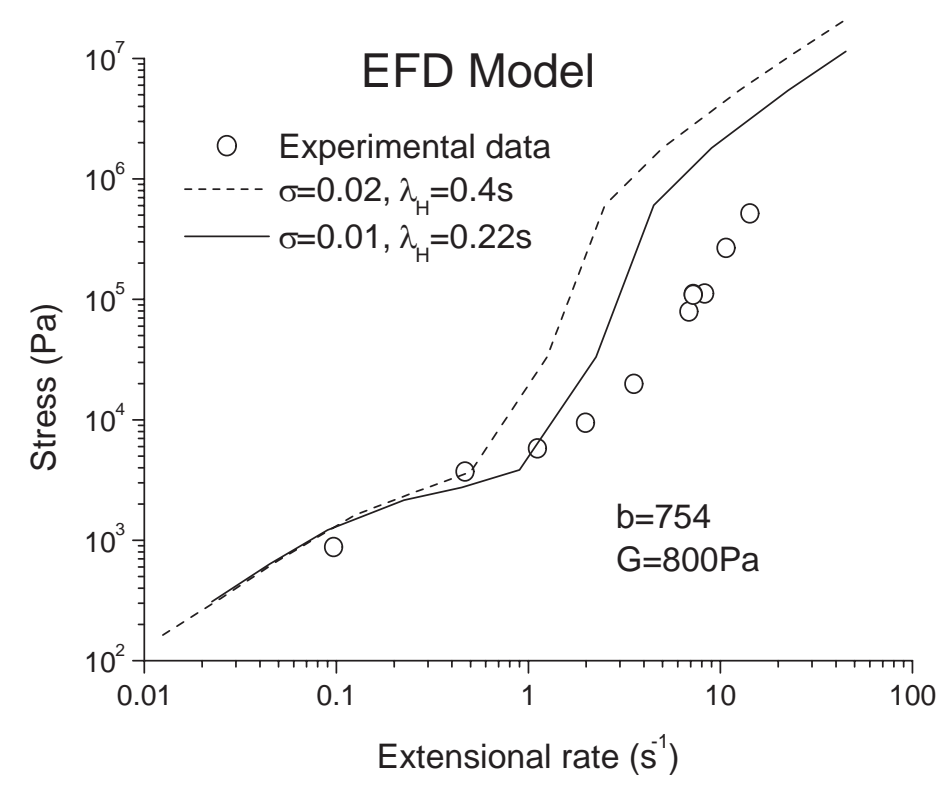

Fig. 9. Steady-state values of the first normal stress difference $\tau_{x x}-\tau_{y y}$ in uniaxial extensional flow. Shown are the predictions of the EFD model and experimental data [3].

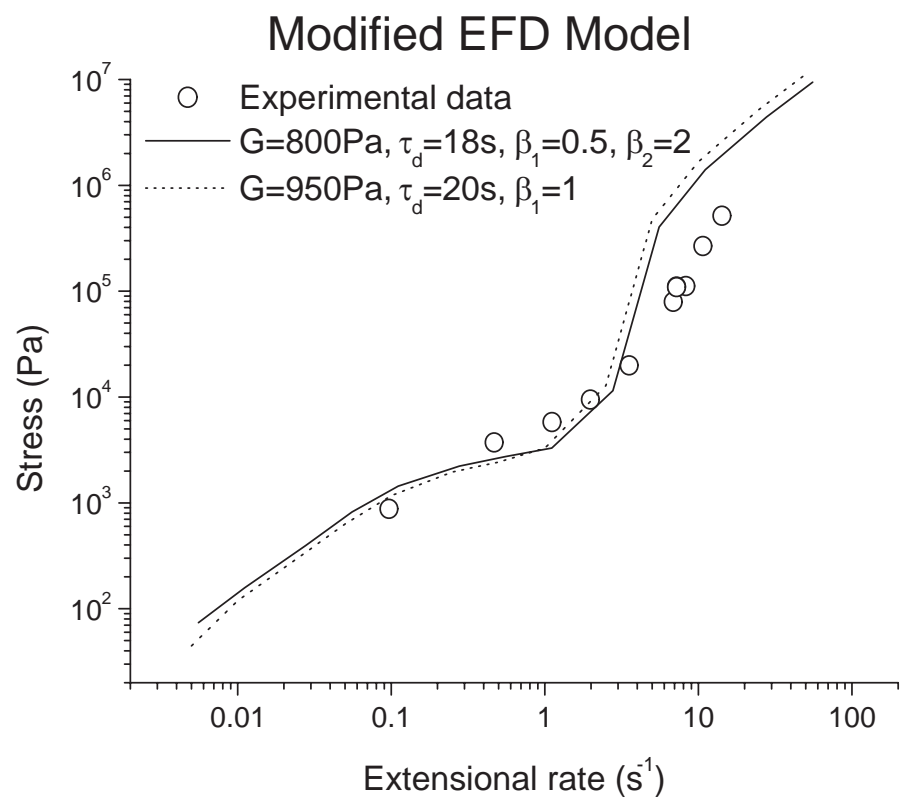

Fig. 10. Steady-state values of the first normal stress difference $\tau_{x x}-\tau_{y y}$ in uniaxial extensional flow. Shown are the predictions of the modified EFD model and experimental data [3]. The dotted curve was generated with the choice of $\beta_{1}=1$ and $\tau_{s}=\tau_{R}$.

with some hope of agreement, would probably require a multi-mode model) a glance at the experimental results of Venerus and Kahvand [40] for a $12 \mathrm{wt} \%$ solution of polystyrene in tricresyl phosphate for the same strains $S_{1}$ and $S_{2}$ (see Figs. 8 and 9 of [40]) indicate that the expected $\tau_{x y}$ overshoot for $t_{w} / \tau_{d}$ sufficiently small fails to materialize in Fig. 11 for the coupled DCR-CS model. 


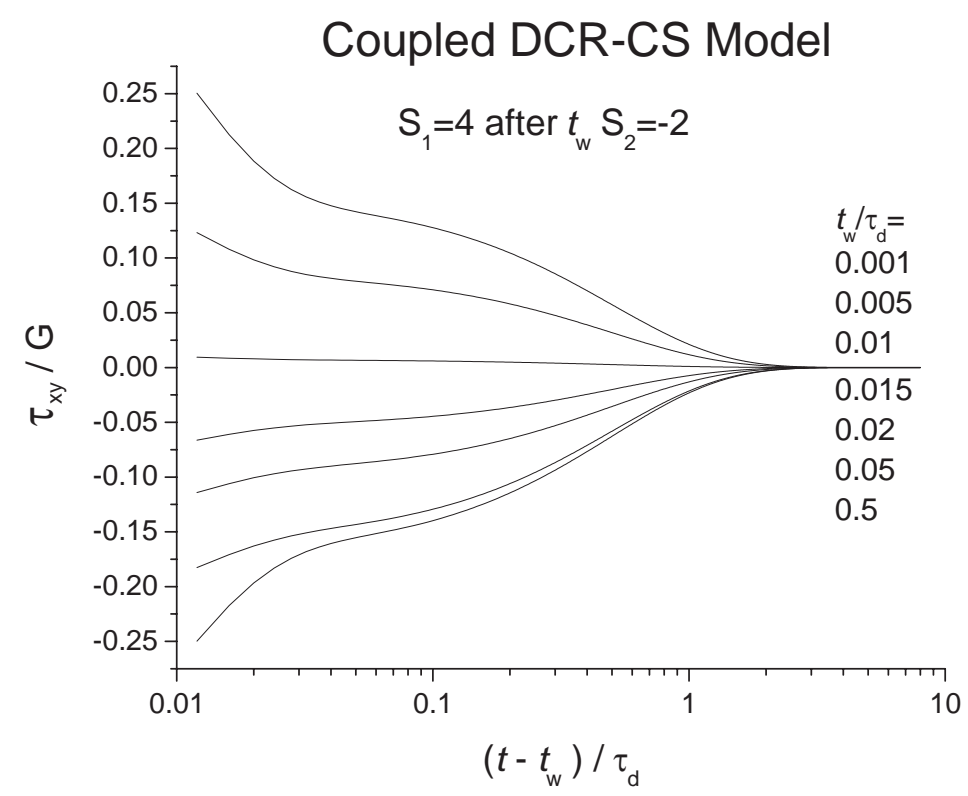

Fig. 11. Relaxation of the normalized shear stress following a reversing double-step strain. Shown are the predictions of the coupled DCR-CS model for differing values of the scaled inter-strain time $t_{w} / \tau_{d}$.

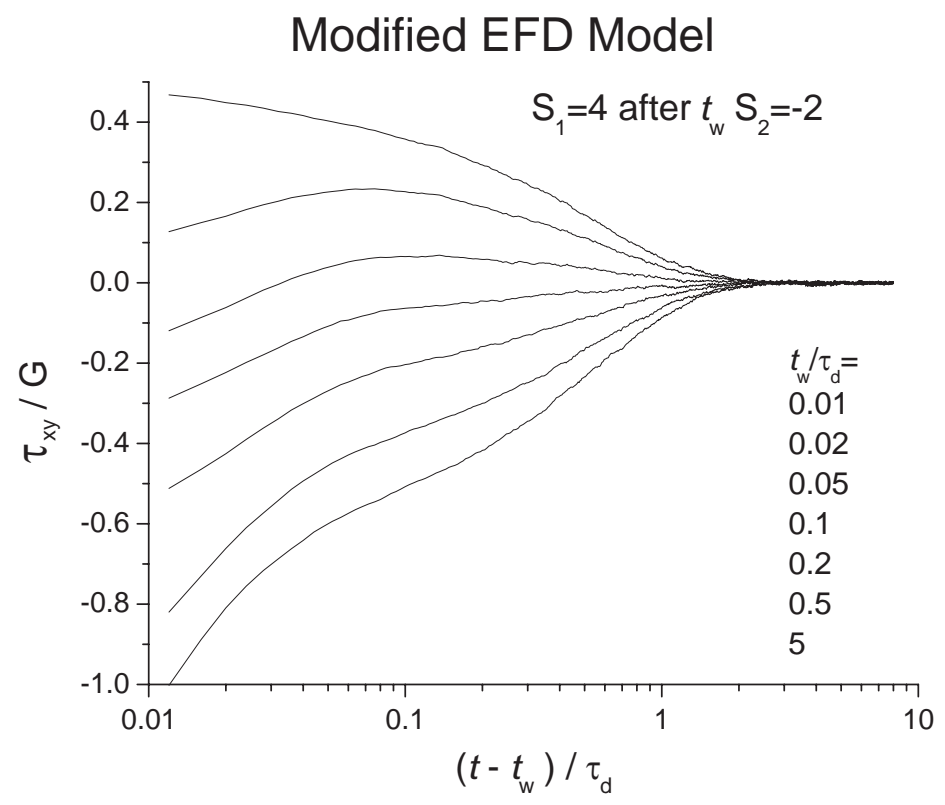

Fig. 12. Relaxation of the normalized shear stress following a reversing double-step strain. Shown are the predictions of the modified EFD model for differing values of the scaled inter-strain time $t_{w} / \tau_{d}$.

Qualitatively, then, the modified EFD model returns superior results to those of the coupled DCR-CS model for reversing double step-strain flow. 


\section{Conclusions}

In this paper we have presented modifications to two models in the literature that describe entangled linear polymers. Firstly, the SU model of Öttinger [34] has been modified to include the effect of irreducible Rouse friction. Second, the EFD model of Bird and Deaguiar [4] has been modified to take into account $\mathrm{CCR}$ and irreducible Rouse friction.

Although both modified models are able to quantitatively describe experimental data in steady shear flows, the coupling of orientation and stretch has been shown to be important in the modified EFD model since this permits the use of realistic values of the maximum stretch ratio. It has been shown that the modified EFD model can, additionally, quantitatively predict rheological properties in steady uniaxial extensional flow and qualitatively capture the transient behaviour of a real concentrated polymer solution in start-up of simple shearing flow and in the reversing double step-strain experiment. When compared with the coupled model of Marrucci and Ianniruberto [27] the modified EFD model is overall quantitatively better at describing the data in steady simple shear flow and uniaxial extensional flow and is qualitatively better in capturing transient behaviour in a reversing double step-strain experiment. Since the modified EFD model is based on a single segment theory and has only 3 degrees of freedom it is a good candidate for complex flow simulations with CONNFFESSIT [22], the Brownian configuration fields method [16,37] or Fokker-Planck-based numerical methods [23].

\section{Acknowledgements}

The work of the second author has been supported by the Swiss National Science Foundation, grant number 2100-55543. The authors wish to thank Martin Kröger for permission to use the DEC-UNIX Cluster of the Polymer Physics Group at the ETH Zürich for the Brownian dynamics simulations. 


\section{References}

[1] M. Bercea, C. Peiti, B. Simionescu and P. Navard, Shear rheology of semidilute poly(methyl methacrylate) solutions, Macromolecules 26 (1993) 7095-7096.

[2] P. K. Bhattacharjee, D. A. Nguyen, G. H. McKinley and T. Sridhar, Extensional stress growth and stress relaxation in entangled polymer solutions, J. Rheol. 47 (2003) 269-290.

[3] P. K. Bhattacharjee, J. P. Oberhauser, G. H. McKinley, L. G. Leal and T. Sridhar, Extensional rheometry of entangled solutions, Macromolecules 35 (2002) 10131-10148.

[4] R. B. Bird and J. R. Deaguiar, An encapsulated dumbbell model for concentrated polymer solutions and melts I. Theoretical developments and constitutive equation, J. Non-Newtonian Fluid Mech. 13 (1983) 149-160.

[5] P. G. de Gennes, Reptation of a polymer chain in the presence of fixed obstacles, J. Chem. Phys. 55 (1971) 572-579.

[6] J. des Cloizeaux, Double reptation vs simple reptation in polymer melts, Europhys. Lett. 5 (1988) 437-442.

[7] M. Doi, A constitutive equation derived from the model of Doi and Edwards for concentrated polymer solutions and polymer melts, J. Polym. Sci. Polym. Phys. Ed. 18 (1980) 2055-2067.

[8] M. Doi, Stress relaxation of polymeric liquids after double step strain, J. Polym. Sci. Polym. Phys. Ed. 18 (1980) 1891-1905.

[9] M. Doi and S. F. Edwards, The Theory of Polymer Dynamics, Clarendon, Oxford, 1986.

[10] J. Fang, Development of Thermodynamically Admissible Molecular-based Models for Entangled Polymers, PhD Thesis, ETH Zürich, 2001.

[11] J. Fang, M. Kröger and H. C. Öttinger, A thermodynamically admissible reptation model for fast flows of entangled polymers. II. Model predictions for shear and extensional flows, J. Rheol. 44 (2000) 1293-1317.

[12] M. Grmela and H. C. Öttinger, Dynamics and thermodynamics of complex fluids. I. Development of a general formalism, Phys. Rev. E 56 (1997) 66206632 .

[13] C. C. Hua and J. D. Schieber, Segment connectivity, chain-length breathing, segmental stretch, and constraint release in reptation models. I. Theory and single-step strain predictions, J. Chem. Phys. 109 (1998) 10018-10027.

[14] C. C. Hua, J. D. Schieber and D. C. Venerus, Segment connectivity, chainlength breathing, segmental stretch, and constraint release in reptation models. II. Double-step strain predictions, J. Chem. Phys. 109 (1998) 10028-10032. 
[15] C. C. Hua, J. D. Schieber and D. C. Venerus, Segment connectivity, chainlength breathing, segmental stretch, and constraint release in reptation models. III. Shear flows, J. Rheol. 43 (1999) 701-717.

[16] M. A. Hulsen, A. P. G. van Heel and B. H. A. A. van den Brule, Simulation of viscoelastic flows using Brownian configuration fields, J. Non-Newtonian Fluid Mech. 70 (1997) 79-101.

[17] G. Ianniruberto and G. Marrucci, On compatibility of the Cox-Merz rule with the model of Doi and Edwards, J. Non-Newtonian Fluid Mech. 65 (1996) 241246.

[18] G. Ianniruberto and G. Marrucci, Convective orientational renewal in entangled polymers, J. Non-Newtonian Fluid Mech. 95 (2000) 363-374.

[19] G. Ianniruberto and G. Marrucci, A simple constitutive equation for entangled polymers with chain stretch, J. Rheol. 45 (2001) 1305-1318.

[20] G. Ianniruberto and G. Marrucci, A multi-mode CCR model for entangled polymers with chain stretch, J. Non-Newtonian Fluid Mech. 102 (2002) 383395.

[21] H. Kahvand, Strain Coupling Effects in Polymer Rheology, PhD Thesis, Illinois Institute of Technology, 1995.

[22] M. Laso and H. C. Öttinger, Calculation of viscoelastic flow using molecular models: the CONNFFESSIT approach, J. Non-Newtonian Fluid Mech. 47 (1993) 1-20.

[23] A. Lozinski, C. Chauvière, J. Fang and R. G. Owens, A Fokker-Planck simulation of fast flows of melts and concentrated polymer solutions in complex geometries, J. Rheol. 47 (2003) 535-561.

[24] G. Marrucci, The Doi-Edwards model without independent alignment, J. NonNewtonian Fluid Mech. 21 (1986) 329-336.

[25] G. Marrucci, Dynamics of entanglements: A nonlinear model consistent with the Cox-Merz rule, J. Non-Newtonian Fluid Mech. 62 (1996) 279-289.

[26] G. Marrucci and N. Grizzuti, Fast flow of concentrated polymers: Predictions of the tube model on chain stretching, Gazz. Chim. Ital. 118 (1988) 179-185.

[27] G. Marrucci and G. Ianniruberto, Flow-induced orientation and stretching of entangled polymers, Phil. Trans. R. Soc. Lond. A 361 (2003) 677-687.

[28] D. W. Mead, R. G. Larson and M. Doi, A molecular theory for fast flows of entangled polymers, Macromolecules 31 (1998) 7895-7914.

[29] D. W. Mead and L. G. Leal, The reptation model with segmental stretch I. Basic equations and general properties, Rheol Acta 34 (1995) 339-359.

[30] D. W. Mead, D. Yavich and L. G. Leal, The reptation model with segmental stretch II. Steady flow properties, Rheol. Acta 34 (1995) 360-383. 
[31] H. C. Öttinger, Modified reptation model, Phys. Rev. E 50 (1994) 4891-4895.

[32] H. C. Öttinger, Stochastic Processes in Polymeric Fluids: Tools and Examples for Developing Simulation Algorithms, Springer, Berlin, 1996.

[33] H. C. Öttinger, General projection operator formalism for the dynamics and thermodynamics of complex fluids, Phys. Rev. E 57 (1998) 1416-1420.

[34] H. C. Öttinger, A thermodynamically admissible reptation model for fast flows of entangled polymers, J. Rheol. 43 (1999) 1461-1493.

[35] H. C. Öttinger and A. N. Beris, Thermodynamically consistent reptation model without independent alignment, J. Chem. Phys. 110 (1999) 6593-6596.

[36] H. C. Öttinger and M. Grmela, Dynamics and thermodynamics of complex fluids. II. Illustrations of a general formalism, Phys. Rev. E 56 (1997) 66336655 .

[37] H. C. Öttinger, B. H. A. A. van den Brule and M. A. Hulsen, Brownian configuration fields and variance reduced CONNFFESSIT, J. Non-Newtonian Fluid Mech. 70 (1997) 255-261.

[38] D. S. Pearson, E. A. Herbolzheimer, G. Marrucci and N. Grizzuti, Transient behavior of entangled polymers at high shear rates, J. Polym. Sci. Polym. Phys. Ed. 29 (1991) 1589-1597.

[39] C. Tsenoglou, Viscoelasticity of binary homopolymer blends, ACS Polym. Preprints 28 (1987) 185-186.

[40] D. C. Venerus and H. Kahvand, Doi-Edwards theory evaluation in double-step strain flows, J. Polym. Sci., Part B: Polym. Phys. 32 (1994) 1531-1542.

[41] J. van Meerveld, Private communication (2002).

[42] P. Wapperom, R. Keunings and G. Ianniruberto, Prediction of rheometrical and complex flows of entangled linear polymers using the double-convectionreptation model with chain stretch, J. Rheol. 47 (2003) 247-265.

[43] P. Wapperom and R. Keunings, Impact of decoupling approximation between stretch and orientation in rheometrical and complex flow of entangled linear polymers, J. Non-Newtonian Fluid Mech. This volume (2004). 